\title{
Cascade trifluoromethylthiolation and cyclization of $\mathbf{N}$-[(3-aryl)propioloyl]indoles
}

\author{
Ming-Xi Bi ${ }^{1}$, Shuai Liu ${ }^{1}$, Yangen Huang ${ }^{1}$, Xiu-Hua X ${ }^{* 2}$ and Feng-Ling Qing ${ }^{* 1,2}$
}

Address:

${ }^{1}$ Key Laboratory of Science and Technology of Eco-Textiles, Ministry of Education, College of Chemistry, Chemical Engineering and Biotechnology, Donghua University, 2999 North Renmin Lu, Shanghai 201620, China and ${ }^{2}$ Key Laboratory of Organofluorine Chemistry, Center for Excellence in Molecular Synthesis, Shanghai Institute of Organic Chemistry, University of Chinese Academy of Science, Chinese Academy of Science, 345 Lingling Lu, Shanghai 200032, China

Email:

Xiu-Hua Xu* - xuxiuhua@sioc.ac.cn; Feng-Ling Qing* -

flq@mail.sioc.ac.cn

* Corresponding author

Keywords:

cyclization; indole derivatives; oxidation; radical reaction;

trifluoromethylthiolation

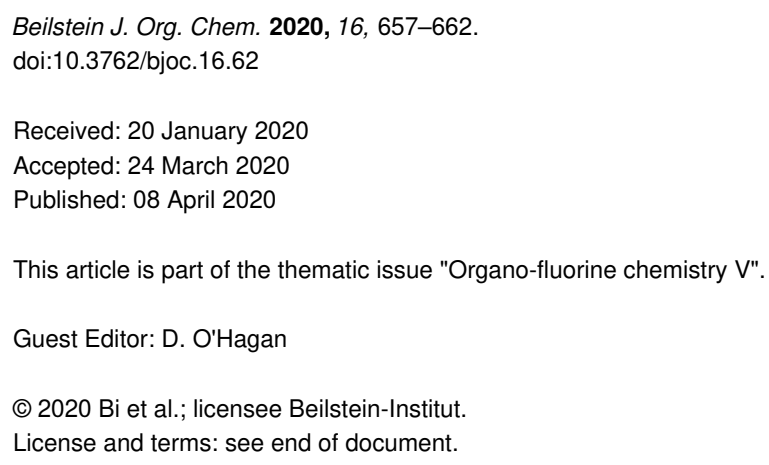


that enable the efficient synthesis of $\mathrm{SCF}_{3}$-containing compounds, especially those featuring medicinally promising scaffolds.

Pyrrolo[1,2- $a$ ]indol-3-ones are prevalent scaffolds that widely exist in many bioactive compounds and natural products [1720]. Representative examples of biologically active pyrrolo[1,2a] indol-3-one derivatives are shown in Figure 1. Recently, the development of efficient methods for the synthesis of pyrrolo[1,2-a]indol-3-one derivatives has attracted considerable attention. For instance, Song [21] and Liang [22] reported the one-pot synthesis of novel phosphorylated and sulfonylated pyrrolo[1,2- $a$ ]indol-3-ones from $N$-[(3-phenyl)propioloyl]indole and $N$-propargylindoles, respectively. Inspired by these elegant results, we became interested in the preparation of $\mathrm{SCF}_{3}$-substituted pyrrolo[1,2- $a$ ]indol-3-ones, which might be potentially useful in medicinal chemistry.<smiles></smiles>

mitosene lactam<smiles>COc1ccc2c(c1)c(CCNC(C)=O)c1n2C(=O)C=C1C</smiles>

analogue of melatonin<smiles>O=C1N2c3ccccc3C(=O)[C@@]2(c2c[nH]c3ccccc23)[C@@H]2O[C@H](CO)[C@@H](O)[C@]12O</smiles>

isatisine $A$

Figure 1: Representative examples of biologically active pyrrolo[1,2a]indol-3-one derivatives.

Radical cascade reactions constitute highly efficient strategies for the construction of compounds with structural diversity and complexity. In 2014, Wang reported the first radical cascade trifluoromethylthiolation and cyclization of activated alkenes (Scheme 1a) [23]. Afterward, Nevado [24], Hopkinson and Glorius [25], Dagousset and Magnier [26], as well as Fu [27] applied this strategy in the synthesis of a series of $\mathrm{CH}_{2} \mathrm{SCF}_{3}-$ substituted heterocycles. For the construction of $\mathrm{SCF}_{3}$-substituted cyclic compounds, normally proper alkynes are chosen as the substrates for cascade reactions [28-32]. In 2015, Wang developed an oxidative radical cyclization of aryl alkynoate esters with $\mathrm{AgSCF}_{3}$ for the synthesis of trifluoromethylthiolated coumarins (Scheme 1b) [28]. In 2016, Liu exploited the tandem trifluoromethylthiolation/cyclization of $\mathrm{N}$-arylpropiolamides to construct the $\mathrm{SCF}_{3}$-substituted spiro[4,5]trienones (Scheme 1c)
[29]. In the same year, Zhang and Chen disclosed the transformation of arylpropynones to $\mathrm{SCF}_{3}$-substituted indenones through the tandem trifluoromethylthiolation/cyclization processes (Scheme 1d) [30]. As part of our continuing research interest in radical trifluoromethylthiolation reactions [33-38], herein we disclose a cascade trifluoromethylthiolation and cyclization of $N$-[(3-aryl)propioloyl]indoles to access $\mathrm{SCF}_{3}-$ substituted pyrrolo[1,2-a]indol-3-ones (Scheme 1e).
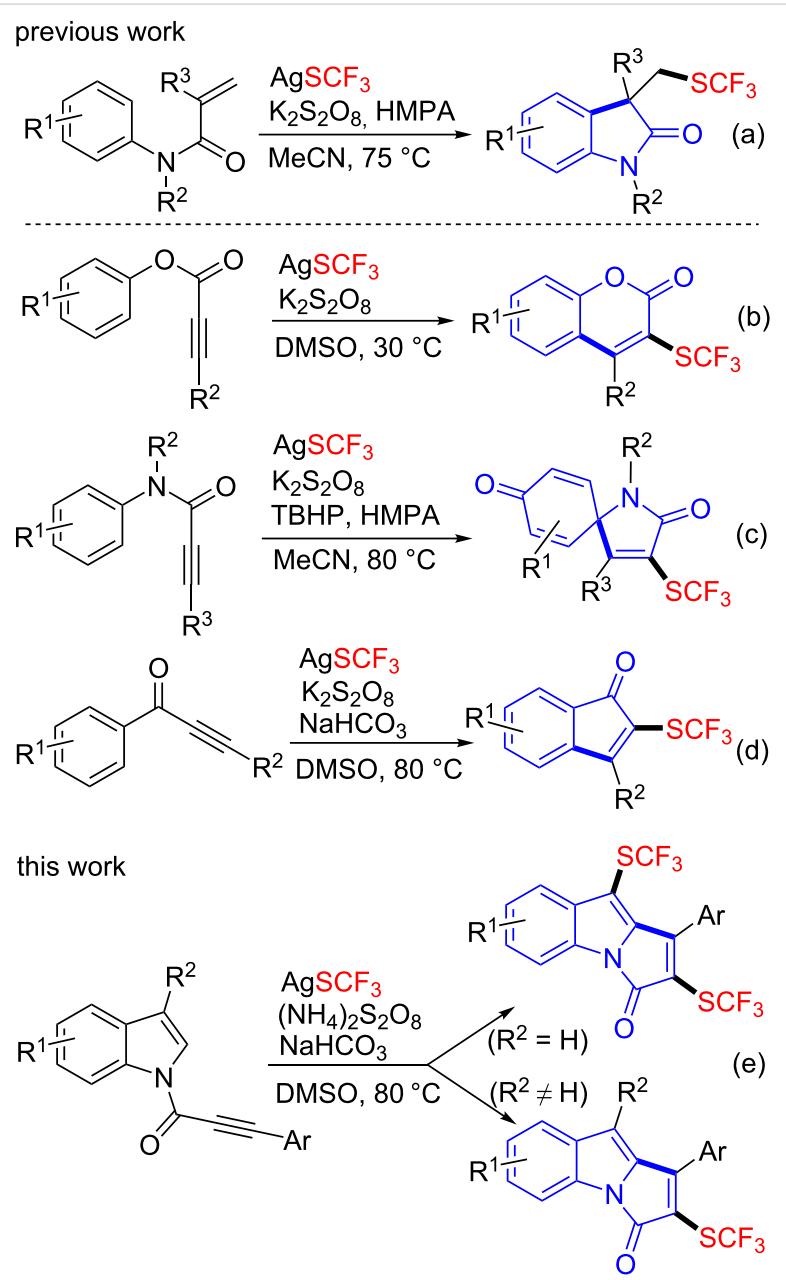

Scheme 1: Radical cascade trifluoromethylthiolation and cyclization reactions.

\section{Results and Discussion}

On the outset, 1-(1H-indol-1-yl)-3-phenylprop-2-yn-1-one (1a) was chosen as the model substrate for optimization of the reaction conditions (Table 1). To our surprise, the reaction of $\mathbf{1 a}$ and $\mathrm{AgSCF}_{3}$ in the presence of $\mathrm{K}_{2} \mathrm{~S}_{2} \mathrm{O}_{8}$ and $\mathrm{KHCO}_{3}$ in DMSO at $80{ }^{\circ} \mathrm{C}$ gave bis(trifluoromethylthiolated) product $2 \mathbf{a}$ in $28 \%$ yield (Table 1, entry 1). Only trace of mono(trifluoromethylthiolated) product was detected, and most of the substrate 1a was not converted. To the best of our knowledge, the combination of bis(trifluoromethylthiolation) [36,39-41] and cascade cycli- 
Table 1: Optimization of the reaction conditions ${ }^{\mathrm{a}}$.

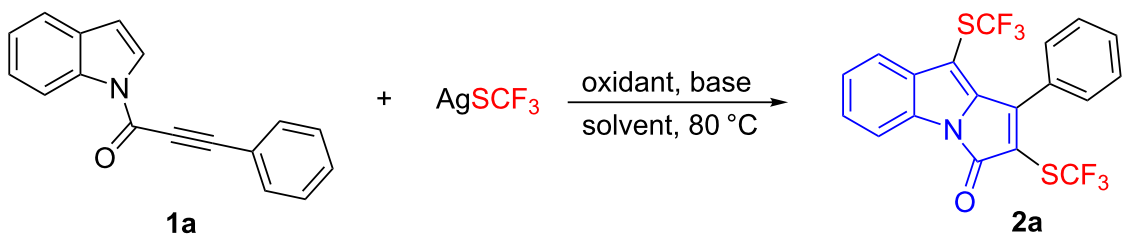

\begin{tabular}{|c|c|c|c|c|}
\hline entry & oxidant & base & solvent & yield $(\%)^{b}$ \\
\hline 1 & $\mathrm{~K}_{2} \mathrm{~S}_{2} \mathrm{O}_{8}$ & $\mathrm{KHCO}_{3}$ & DMSO & 28 \\
\hline $2^{c}$ & $\mathrm{~K}_{2} \mathrm{~S}_{2} \mathrm{O}_{8}$ & $\mathrm{KHCO}_{3}$ & DMSO & 52 \\
\hline $3^{c}$ & $\mathrm{Na}_{2} \mathrm{~S}_{2} \mathrm{O}_{8}$ & $\mathrm{KHCO}_{3}$ & DMSO & 55 \\
\hline $4^{c}$ & $\left(\mathrm{NH}_{4}\right)_{2} \mathrm{~S}_{2} \mathrm{O}_{8}$ & $\mathrm{KHCO}_{3}$ & DMSO & 58 \\
\hline $5^{c}$ & $\left(\mathrm{NH}_{4}\right)_{2} \mathrm{~S}_{2} \mathrm{O}_{8}$ & $\mathrm{~K}_{2} \mathrm{CO}_{3}$ & DMSO & 40 \\
\hline $6^{c}$ & $\left(\mathrm{NH}_{4}\right)_{2} \mathrm{~S}_{2} \mathrm{O}_{8}$ & $\mathrm{NaHCO}_{3}$ & DMSO & 72 \\
\hline $7^{c}$ & $\left(\mathrm{NH}_{4}\right)_{2} \mathrm{~S}_{2} \mathrm{O}_{8}$ & $\mathrm{DBU}$ & DMSO & 34 \\
\hline $8^{c}$ & $\left(\mathrm{NH}_{4}\right)_{2} \mathrm{~S}_{2} \mathrm{O}_{8}$ & $\mathrm{NaHCO}_{3}$ & $\mathrm{MeCN}$ & 8 \\
\hline $9^{c}$ & $\left(\mathrm{NH}_{4}\right)_{2} \mathrm{~S}_{2} \mathrm{O}_{8}$ & $\mathrm{NaHCO}_{3}$ & DMF & trace \\
\hline $10^{d}$ & $\left(\mathrm{NH}_{4}\right)_{2} \mathrm{~S}_{2} \mathrm{O}_{8}$ & $\mathrm{NaHCO}_{3}$ & DMSO & 80 \\
\hline
\end{tabular}


mined by ${ }^{19} \mathrm{~F}$ NMR using trifluorotoluene as an internal standard. ${ }^{\mathrm{C}} \mathrm{AgSCF}_{3}(0.3 \mathrm{mmol})$, oxidant $(0.3 \mathrm{mmol}) .{ }^{\mathrm{d}} \mathrm{AgSCF}_{3}(0.3 \mathrm{mmol})$, oxidant $(0.3 \mathrm{mmol})$, base $(0.1 \mathrm{mmol})$.

zation reactions has not been reported before. Thus, the amounts of $\mathrm{AgSCF}_{3}$ and $\mathrm{K}_{2} \mathrm{~S}_{2} \mathrm{O}_{8}$ were increased to deliver 2a in $52 \%$ yield (Table 1 , entry 2 ). Other oxidants including $\mathrm{Na}_{2} \mathrm{~S}_{2} \mathrm{O}_{8}$ and $\left(\mathrm{NH}_{4}\right)_{2} \mathrm{~S}_{2} \mathrm{O}_{8}$ afforded $2 \mathbf{a}$ in slightly higher yields, respectively (Table 1, entries 3 and 4). Switching $\mathrm{KHCO}_{3}$ to $\mathrm{NaHCO}_{3}$ could enhance the yield (Table 1, entry 6), whereas $\mathrm{K}_{2} \mathrm{CO}_{3}$ and DBU reduced the reaction efficiency (Table 1, entries 5 and 7). Subsequent evaluation of solvents revealed that $\mathrm{MeCN}$ and DMF were inferior to DMSO (Table 1, entries 8 and 9). Gratifyingly, the yield was improved to $80 \%$ by reducing the amount of base to 1.0 equivalent (Table 1, entry 10).

With the optimized reaction conditions in hand, we then set out to explore the substrate scope of $N$-[(3-aryl)propioloyl]indoles (Scheme 2). First, we explored the effect of the substitution on the indole ring. Both electron-donating and withdrawing groups at different positions of the indole ring produced the bis(trifluoromethylthiolated) products $\mathbf{2 a - 0}$ in moderate to good yields. A wide range of functionalities such as alkyl, alkoxy, nitro, nitrile, ester, aldehyde, fluoro, chloro, and bromo were well-tolerated and compatible under the mild reaction conditions. Substrate 1p containing a methyl substituent on the phenyl ring could also participate in the reaction and furnish the desired product in moderate yield. However, attempts to prepare the substrates bearing an alkyl or electron-deficient aryl substituent on the alkynone were not successful. The structure of product $\mathbf{2 a}$ was unambiguously identified by single-crystal X-ray analysis.
When the $N$-[(3-aryl)propioloyl]indole substrates (3a-d) with different substituents at the 3-position of the indole ring were subjected to the standard conditions, the cascade trifluoromethylthiolation and cyclization occurred to yield trifluoromethylthiolated pyrrolo[1,2-a]indol-3-ones (4a-d) in moderate yields (Scheme 3 ). The functionalities including alkyl, aryl, nitrile, and acyl were also well tolerated in this reaction.

In order to gain insight into the reaction mechanism, the radical scavenger 2,2,6,6-tetramethylpiperidin-1-oxyl (TEMPO) was added to the standard reactions of $\mathbf{1 a}$ and $\mathbf{3 b}$, respectively. The desired product $\mathbf{2 a}$ was not formed and only trace of $\mathbf{4 b}$ was detected (see the Supporting Information File 1), which suggested that the radical process was probably involved in these transformations. Notably, no TEMPO-trapped product was detected by ${ }^{19} \mathrm{~F}$ NMR spectra of the crude reaction mixtures. On the basis of these results and literature studies [21,23,42-47], a plausible reaction mechanism was proposed in Scheme 4. First, oxidation of $\mathrm{AgSCF}_{3}$ by $\left(\mathrm{NH}_{4}\right)_{2} \mathrm{~S}_{2} \mathrm{O}_{8}$ generates $\mathrm{Ag}^{\mathrm{II}} \mathrm{SCF}_{3}$, which could be further transformed to the $\mathrm{CF}_{3} \mathrm{~S}$ radical or $\mathrm{CF}_{3} \mathrm{SSCF}_{3}[23,36]$. Then, the addition of a $\mathrm{CF}_{3} \mathrm{~S}$ radical to the alkyne function of substrates $\mathbf{1}$ or $\mathbf{3}$ afforded intermediate $\mathbf{A}$. Subsequently, cyclization of intermediate $\mathbf{A}$, followed by oxidation with $\left(\mathrm{NH}_{4}\right)_{2} \mathrm{~S}_{2} \mathrm{O}_{8}$, gave intermediate $\mathbf{C}$ [21,42-47]. Finally, deprotonation of intermediate $\mathbf{C}\left(\mathrm{R}^{2} \neq \mathrm{H}\right)$ with $\mathrm{NaHCO}_{3}$ delivered the aromatized product 4 . In the case of intermediate $\mathbf{C}$ $\left(\mathrm{R}^{2}=\mathrm{H}\right)$, intermediate $\mathbf{D}$ was probably formed, and further 


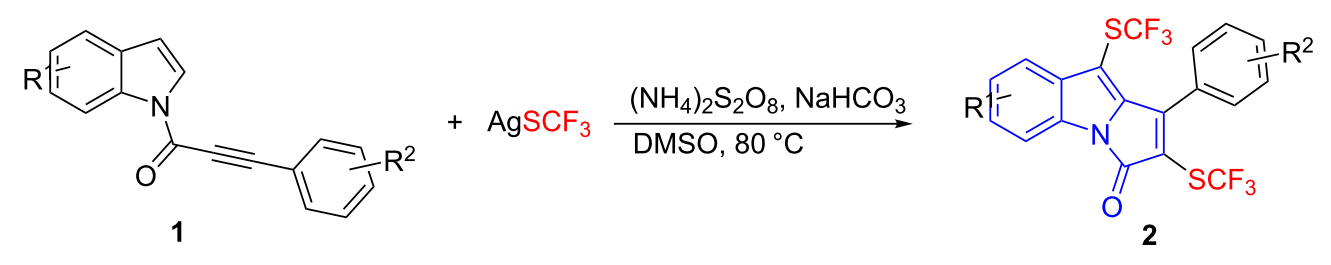<smiles>O=C1C(=O)n2c(c(C(F)(F)F)c3ccccc32)C(c2ccccc2)=C1[Si](F)(F)F</smiles>

2a, $72 \%$

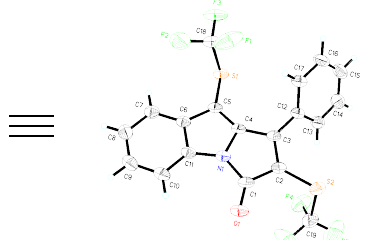

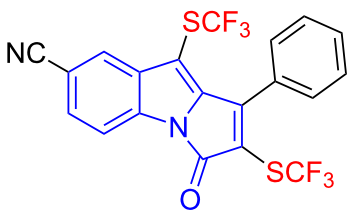

2d, $57 \%$<smiles>COc1ccc2c(c1)c([Se-])c1n2C(=O)C(C(F)(F)F)=C1c1ccccc1</smiles>

2b, $71 \%$<smiles>O=C1C(=O)n2c(c(C(F)(F)F)c3cc([N+](=O)[O-])ccc32)C(c2ccccc2)=C1SC(F)(F)F</smiles><smiles>COC(=O)c1ccc2c(c1)c(C(F)(F)F)c1n2C(=O)C(C(F)(F)F)=C1c1ccccc1</smiles>

2e, $64 \%$<smiles>O=C1C([Se]C(F)(F)F)=C(c2ccccc2)c2c([As])c3cc(F)ccc3n21</smiles>

2g, $68 \%$<smiles></smiles>

2h, $70 \%$<smiles>O=C1C([Se-](F)(F)F)=C(c2ccccc2)c2c(C(F)(F)F)c3cc(Br)ccc3n21</smiles>

2i, $73 \%$<smiles>Cc1cccc2c1c(C(F)(F)F)c1n2C(=O)C(C(F)(F)F)=C1c1ccccc1</smiles>

2j, $47 \%$<smiles>COc1cccc2c1c(C(F)(F)F)c1n2C(=O)C(C(F)(F)F)=C1c1ccccc1</smiles>

2k, $44 \%$<smiles>CCOC(=O)c1cccc2c1c(C(F)(F)F)c1n2C(=O)C(C(F)(F)F)=C1c1ccccc1</smiles>

2I, $59 \%$<smiles>Cc1ccc2c(C(F)(F)F)c3n(c2c1)C(=O)C(C(F)(F)F)=C3c1ccccc1</smiles>

2m, $45 \%$<smiles>COc1ccc2c(C(F)(F)F)c3n(c2c1)C(=O)C(C(F)(F)F)=C3c1ccccc1</smiles>

2n, $35 \%$<smiles>CC(C)(F)C1=C(C(F)(F)F)c2c(C(F)(F)F)c3ccc(Cl)cc3n2C1=O</smiles>

2o, $61 \%$<smiles>Cc1ccc(C2=C(C(F)(F)F)C(=O)n3c2c(C(F)(F)F)c2ccccc23)cc1</smiles>

2p, $62 \%$

Scheme 2: Cascade bis(trifluoromethylthiolation) and cyclization of $N$-[(3-aryl)propioloyl]indoles 1. Reaction conditions: 1 (0.25 mmol), AgSCF 3 $(0.75 \mathrm{mmol}),\left(\mathrm{NH}_{4}\right)_{2} \mathrm{~S}_{2} \mathrm{O}_{8}(0.75 \mathrm{mmol}), \mathrm{NaHCO}_{3}(0.25 \mathrm{mmol}), \mathrm{DMSO}(5.0 \mathrm{~mL}), 80^{\circ} \mathrm{C}, 12 \mathrm{~h}$, isolated yields.

underwent electrophilic trifluoromethylthiolation with $\mathrm{CF}_{3} \mathrm{SSCF}_{3}[48,49]$ to furnish the bis(trifluoromethylthiolated) product 2.

\section{Conclusion}

We have reported the cascade trifluoromethylthiolation and cyclization reactions for the preparation of novel and poten- tially useful $\mathrm{SCF}_{3}$-containing pyrrolo[1,2- $a$ ]indol-3-ones. Oxidative trifluoromethylthiolation of $N$-[(3-aryl)propioloyl]indoles without substituent at the 3-position of the indole ring with $\mathrm{AgSCF}_{3}$ afforded the bis(trifluoromethylthiolated) products in moderate to good yields, whereas the substrates with a substituent at the 3-position of the indole ring were converted to the mono(trifluoromethylthiolated) products in moderate yields. 

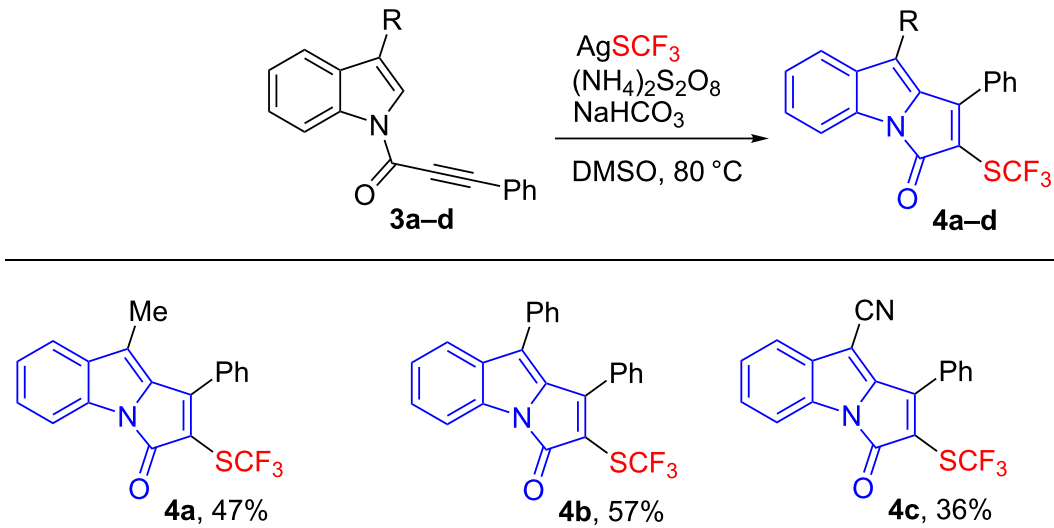<smiles>CS(F)(F)(F)(F)C1=C(c2ccccc2)c2c(C#N)c3ccccc3n2C1=O</smiles><smiles>CC(C)(C)C1=C(c2ccccc2)C(c2ccccc2)=C(C(F)(F)F)C1=O</smiles>

Scheme 3: Cascade trifluoromethylthiolation and cyclization of $N$-[(3-aryl)propioloyl]indoles 3. Reaction conditions: 3 (0.25 mmol), AgSCF 3 $(0.75 \mathrm{mmol}),\left(\mathrm{NH}_{4}\right)_{2} \mathrm{~S}_{2} \mathrm{O}_{8}(0.75 \mathrm{mmol}), \mathrm{NaHCO}_{3}(0.25 \mathrm{mmol}), \mathrm{DMSO}(5.0 \mathrm{~mL}), 80^{\circ} \mathrm{C}, 12 \mathrm{~h}$, isolated yields.<smiles>[R]c1cn(C(=O)C#CC)c2ccccc12</smiles>

1 or 3<smiles>[R]c1cn(C(=O)C(=C[Al])SC(F)(F)F)c2ccc(O)cc12</smiles>

A

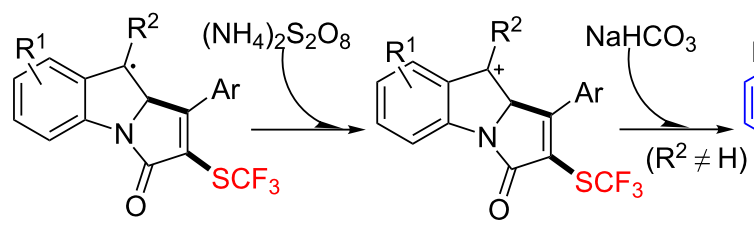

C

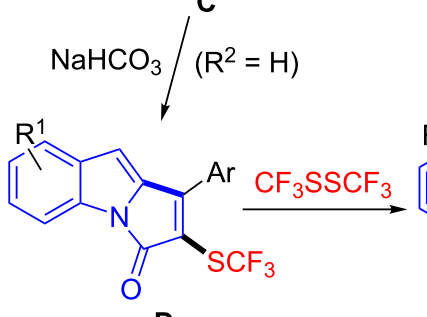

D<smiles>[R]c1c2n(c3ccccc13)C(=O)C(SC(F)(F)F)=C2[Al]</smiles>

4<smiles>O=c1c(SC(F)(F)F)c(Br)c2c(SC(F)(F)F)c3c[R]cccc3n12</smiles>

Scheme 4: Proposed reaction mechanism.

Further studies on applying radical cascade reactions to the construction of fluorine-containing heterocyclic scaffolds are in progress in our laboratory.

\section{Supporting Information}

\section{Supporting Information File 1}

Experimental procedures, spectroscopic and X-ray data (CCDC 1968129 for compound 2a) and copies of NMR spectra.

[https://www.beilstein-journals.org/bjoc/content/ supplementary/1860-5397-16-62-S1.pdf]

\section{Funding}

National Natural Science Foundation of China (21991121, 21421002), the Strategic Priority Research Program of the Chinese Academy of Sciences (XDB20000000), and Youth
Innovation Promotion Association CAS (No. 2016234) are greatly acknowledged for funding this work.

\section{ORCID ${ }^{\circledR}$ iDs}

Xiu-Hua Xu - https://orcid.org/0000-0002-0759-2286

\section{References}

1. Hansch, C.; Leo, A.; Unger, S. H.; Kim, K. H.; Nikaitani, D.; Lien, E. J. J. Med. Chem. 1973, 16, 1207-1216. doi:10.1021/jm00269a003

2. Hansch, C.; Leo, A.; Taft, R. W. Chem. Rev. 1991, 91, 165-195. doi:10.1021/cr00002a004

3. Purser, S.; Moore, P. R.; Swallow, S.; Gouverneur, V. Chem. Soc. Rev. 2008, 37, 320-330. doi:10.1039/b610213c

4. Wang, J.; Sánchez-Roselló, M.; Aceña, J. L.; del Pozo, C.; Sorochinsky, A. E.; Fustero, S.; Soloshonok, V. A.; Liu, H. Chem. Rev. 2014, 114, 2432-2506. doi:10.1021/cr4002879

5. Meanwell, N. A. J. Med. Chem. 2018, 61, 5822-5880. doi:10.1021/acs.jmedchem.7b01788

6. Leroux, F.; Jeschke, P.; Schlosser, M. Chem. Rev. 2005, 105, 827-856. doi:10.1021/cr040075b 
7. Boiko, V. N. Beilstein J. Org. Chem. 2010, 6, 880-921. doi:10.3762/bjoc.6.88

8. Manteau, B.; Pazenok, S.; Vors, J.-P.; Leroux, F. R. J. Fluorine Chem. 2010, 131, 140-158. doi:10.1016/j.jfluchem.2009.09.009

9. Chu, L.; Qing, F.-L. Acc. Chem. Res. 2014, 47, 1513-1522. doi:10.1021/ar4003202

10. Toulgoat, F.; Alazet, S.; Billard, T. Eur. J. Org. Chem. 2014, 2415-2428. doi:10.1002/ejoc.201301857

11. Shao, X.; Xu, C.; Lu, L.; Shen, Q. Acc. Chem. Res. 2015, 48, 1227-1236. doi:10.1021/acs.accounts.5b00047

12. Xu, X.-H.; Matsuzaki, K.; Shibata, N. Chem. Rev. 2015, 115, 731-764. doi:10.1021/cr500193b

13. Chachignon, H.; Cahard, D. Chin. J. Chem. 2016, 34, 445-454. doi:10.1002/cjoc.201500890

14. Barata-Vallejo, S.; Bonesi, S.; Postigo, A. Org. Biomol. Chem. 2016, 14, 7150-7182. doi:10.1039/c6ob00763e

15. Zheng, H.; Huang, Y.; Weng, Z. Tetrahedron Lett. 2016, 57, 1397-1409. doi:10.1016/j.tetlet.2016.02.073

16. Hardy, M. A.; Chachignon, H.; Cahard, D. Asian J. Org. Chem. 2019, 8, 591-609. doi:10.1002/ajoc.201900004

17. Toyota, M.; Ihara, M. Nat. Prod. Rep. 1998, 15, 327-340. doi:10.1039/a815327y

18. Elmegeed, G. A.; Baiuomy, A. R.; Abdel-Salam, O. M. E. Eur. J. Med. Chem. 2007, 42, 1285-1292. doi:10.1016/j.ejmech.2007.01.027

19. Liu, J.-F.; Jiang, Z.-Y.; Wang, R.-R.; Zheng, Y.-T.; Chen, J.-J.; Zhang, X.-M.; Ma, Y.-B. Org. Lett. 2007, 9, 4127-4129. doi:10.1021/ol701540y

20. Bass, P. D.; Gubler, D. A.; Judd, T. C.; Williams, R. M. Chem. Rev. 2013, 113, 6816-6863. doi:10.1021/cr3001059

21. Xu, J.; Yu, X.; Song, Q. Org. Lett. 2017, 19, 980-983. doi:10.1021/acs.orglett.6b03713

22. Zhu, X.-Y.; Han, Y.-P.; Li, M.; Li, X.-S.; Liang, Y.-M. Adv. Synth. Catal. 2018, 360, 3460-3465. doi:10.1002/adsc.201800414

23. Yin, F.; Wang, X.-S. Org. Lett. 2014, 16, 1128-1131. doi:10.1021/ol403739w

24. Fuentes, N.; Kong, W.; Fernández-Sánchez, L.; Merino, E.; Nevado, C. J. Am. Chem. Soc. 2015, 137, 964-973. doi:10.1021/ja5115858

25. Honeker, R.; Garza-Sanchez, R. A.; Hopkinson, M. N.; Glorius, F. Chem. - Eur. J. 2016, 22, 4395-4399. doi:10.1002/chem.201600190

26. Dagousset, G.; Simon, C.; Anselmi, E.; Tuccio, B.; Billard, T.; Magnier, E. Chem. - Eur. J. 2017, 23, 4282-4286. doi:10.1002/chem.201700734

27. Zhu, M.; Fu, W.; Guo, W.; Tian, Y.; Wang, Z.; Ji, B. Org. Biomol. Chem. 2019, 17, 3374-3380. doi:10.1039/c9ob00342h

28. Zeng, Y.-F.; Tan, D.-H.; Chen, Y.; Lv, W.-X.; Liu, X.-G.; Li, Q.; Wang, H. Org. Chem. Front. 2015, 2, 1511-1515. doi:10.1039/c5qo00271k

29. Jin, D.-P.; Gao, P.; Chen, D.-Q.; Chen, S.; Wang, J.; Liu, X.-Y.; Liang, Y.-M. Org. Lett. 2016, 18, 3486-3489. doi:10.1021/acs.orglett.6b01702

30. Song, Y.-K.; Qian, P.-C.; Chen, F.; Deng, C.-L.; Zhang, X.-G. Tetrahedron 2016, 72, 7589-7593. doi:10.1016/j.tet.2016.10.013

31. Qiu, Y.-F.; Zhu, X.-Y.; Li, Y.-X.; He, Y.-T.; Yang, F.; Wang, J.; Hua, H.-L.; Zheng, L.; Wang, L.-C.; Liu, X.-Y.; Liang, Y.-M. Org. Lett. 2015, 17, 3694-3697. doi:10.1021/acs.orglett.5b01657

32. Qiu, Y.-F.; Niu, Y.-J.; Wei, X.; Cao, B.-Q.; Wang, X.-C.; Quan, Z.-J. J. Org. Chem. 2019, 84, 4165-4178. doi:10.1021/acs.joc.9b00181

33. Zhang, K.; Liu, J.-B.; Qing, F.-L. Chem. Commun. 2014, 50, 14157-14160. doi:10.1039/c4cc07062c
34. Pan, S.; Huang, Y.; Qing, F.-L. Chem. - Asian J. 2016, 11, 2854-2858. doi:10.1002/asia.201601098

35. Li, H.; Liu, S.; Huang, Y.; Xu, X.-H.; Qing, F.-L. Chem. Commun. 2017, 53, 10136-10139. doi:10.1039/c7cc06232j

36. Pan, S.; Li, H.; Huang, Y.; Xu, X.-H.; Qing, F.-L. Org. Lett. 2017, 19, 3247-3250. doi:10.1021/acs.orglett.7b01366

37. Pan, S.; Huang, Y.; Xu, X.-H.; Qing, F.-L. Org. Lett. 2017, 19, 4624-4627. doi:10.1021/acs.orglett.7b02249

38. Ouyang, Y.; Xu, X.-H.; Qing, F.-L. Angew. Chem., Int. Ed. 2019, 58, 18508-18512. doi:10.1002/anie.201911323

39. Tlili, A.; Alazet, S.; Glenadel, Q.; Billard, T. Chem. - Eur. J. 2016, 22, 10230-10234. doi:10.1002/chem.201601338

40. Mesgar, M.; Daugulis, O. Org. Lett. 2017, 19, 4247-4250. doi:10.1021/acs.orglett.7b01901

41. Liu, Y.-L.; Qing, F.-L.; Xu, X.-H. Eur. J. Org. Chem. 2020, 1015-1018. doi:10.1002/ejoc.201901836

42. Zhang, H.; Li, W.; Zhu, C. J. Org. Chem. 2017, 82, 2199-2204. doi:10.1021/acs.joc.6b02673

43. Zhu, X.-Y.; Li, M.; Han, Y.-P.; Chen, S.; Li, X.-S.; Liang, Y.-M. J. Org. Chem. 2017, 82, 8761-8768. doi:10.1021/acs.joc.7b01497

44. Zhang, P.; Gao, Y.; Chen, S.; Tang, G.; Zhao, Y. Org. Chem. Front. 2017, 4, 1350-1353. doi:10.1039/c7qo00167c

45. Chen, H.; Liu, M.; Qiu, G.; Wu, J. Adv. Synth. Catal. 2019, 361, 146-150. doi:10.1002/adsc.201801038

46. Sun, K.; Chen, X.-L.; Zhang, Y.-L.; Li, K.; Huang, X.-Q.; Peng, Y.-Y.; Qu, L.-B.; Yu, B. Chem. Commun. 2019, 55, 12615-12618. doi:10.1039/c9cc06924k

47. Gharpure, S. J.; Shelke, Y. G. Org. Lett. 2017, 19, 5022-5025. doi:10.1021/acs.orglett.7b02005

48. Ma, L.; Cheng, X.-F.; Li, Y.; Wang, X.-S. Tetrahedron Lett. 2016, 57, 2972-2975. doi:10.1016/j.tetlet.2016.05.086

49. Chachignon, H.; Maeno, M.; Kondo, H.; Shibata, N.; Cahard, D. Org. Lett. 2016, 18, 2467-2470. doi:10.1021/acs.orglett.6b01026

\section{License and Terms}

This is an Open Access article under the terms of the Creative Commons Attribution License (http://creativecommons.org/licenses/by/4.0). Please note that the reuse, redistribution and reproduction in particular requires that the authors and source are credited.

The license is subject to the Beilstein Journal of Organic Chemistry terms and conditions:

(https://www.beilstein-journals.org/bjoc)

The definitive version of this article is the electronic one which can be found at: $\underline{\text { doi: } 10.3762 / \text { bjoc. } 16.62}$ 